\title{
BIOECOLOGÍA DE LA RAYA DE AGUA DULCE Potamotrygon magdalenae (Duméril, 1865) (MYLIOBATIFORMES) EN LA CIÉNAGA DE SABAYO, GUAIMARAL, COLOMBIA
}

\section{BIOECOLOGY OF THE FRESHWATER STINGRAY Potamotrygon magdalenae (Duméril, 1865) (MYLIOBATIFORMES) FROM THE CIÉNAGA DE SABAYO, GUAIMARAL, COLOMBIA}

\author{
Herly Bibiana Ramos-Socha1, Marcela Grijalba-Bendeck²
}

\begin{abstract}
1 Bióloga Marina, heraso43@gmail.com² Bióloga Marina, Profesora Asociada Programa de Biología Marina marcela.grijalba@ utadeo.edu.co. Facultad de Ciencias Naturales e Ingeniería, Departamento de Ciencias Biológicas y Ambientales, Programa de Biología Marina, Universidad de Bogotá Jorge Tadeo Lozano. Edificio Mundo Marino, Carrera 2 No. 11-68, Rodadero-Santa Marta, Magdalena, Colombia.
\end{abstract}

Rev. U.D.C.A Act. E Div. Cient. 14(2): 109 - 118, 2011

\section{RESUMEN}

A pesar de la representatividad de Potamotrygon magdalenae en Colombia, por encontrarse a lo largo de la cuenca del río Magdalena y sus afluentes, esta raya no ha sido objeto de estudios previos, desconociéndose la mayor parte de sus aspectos bio-ecológicos. Actualmente son capturadas, incidentalmente, por la pesca artesanal que se efectúa en la Ciénaga de Sabayo (Guaimaral, Magdalena), donde no se les da ningún aprovechamiento. Se examinaron 488 individuos de $P$. magdalenae, capturadas entre octubre 2007 y mayo 2008, empleando chinchorro y trasmallo. Se estimó su captura por unidad de esfuerzo en 1,43 $\pm 0,37$ ind $/ \mathrm{h}$, faena para trasmallo y 59,30 $\pm 9,00 \mathrm{ind} / \mathrm{h}$, para chinchorro y una biomasa $0,38 \pm 0,06 \mathrm{~kg} / \mathrm{h}$ y $81,70 \pm 13,30 \mathrm{~kg} / \mathrm{h}$, respectivamente. La talla media de madurez fue $240 \mathrm{~mm}$ ancho de disco en hembras y 202 en machos; la talla mínima de madurez, se estimó en 164, hembras y 160, machos. El ovario derecho en hembras, se registró con menor desarrollo que el izquierdo, ambos úteros fueron funcionales, conteniendo entre 1 y 5 embriones, los cuales, nacen con tallas entre 87 y $95 \mathrm{~mm}$ AD. Se registraron 39 estómagos vacíos $(14,6 \%)$ y 227 con algún contenido (85,3\%), siendo Polymitarcidae $(55,6 \%)$ y sus larvas $(30,8 \%)$ las de mayor representatividad, indicando que $P$. magdalenae es una especie bentónica insectívora detritívora.
Palabras clave: Potamotrygonidae, raya dulceacuícola, biología, pesquerías, Magdalena, Colombia.

\section{SUMMARY}

One of the most abundant fishes in Colombia is Potamotrygon magdalenae, from the Magdalena River and its tributaries, however, no previous studies on this species exist, unknowing therefore the main bio-ecological aspects. No commercial interest exists on this ray that is caught as bycatch by artesian fishery at Ciénaga de Sabayo (Guaimaral, Magdalena). Between October (2007) and May (2008) 488 individuals of $P$. magdalenae were captured using gillnet and trawl net. The catch per unit effort was calculated as $1.43 \pm 0.37 \mathrm{ind} / \mathrm{h}$ for gillnet and $59.30 \pm 9.00 \mathrm{ind} / \mathrm{h}$ for trawl net, and for biomass estimation $0.38 \pm 0.06 \mathrm{~kg} / \mathrm{h}$ and $81.70 \pm 13.30 \mathrm{~kg} / \mathrm{h}$, respectively. Medium maturity size was estimated as $240 \mathrm{~mm}$ disk width for females and $202 \mathrm{~mm}$ for males, while minimum maturity size was $164 \mathrm{~mm}$ (females) and $160 \mathrm{~mm}$ (males). The right ovary of females was less developed than the left one, both uterus were functional containing 1 to 5 embryos, which birth size was estimated between 87 and 95mm AD. A total of 39 empty stomachs were registered $(14,6 \%)$, and food content was observed on 227 (85,3\%). Polymitarcidae (55,6\%) and its larvae (30,8\%) 
were the most important preys, indicating that $P$. magdalenae is an insectivore and detritivore benthonic species.

Key words: Potamotrygonidae, freshwater stingray, biology, fishery, Magdalena, Colombia.

\section{INTRODUCCIÓN}

Según Nelson (2006), los Myliobatiformes incluyen, entre otras, a la familia Potamotrygonidae Garman, 1977; de los géneros que allí están contenidos, Paratrygon y Potamotrygon son los únicos representados en aguas colombianas (Puentes et al. 2009). Estos abarcan siete especies, cuatro de ellas (Potamotrygon motoro, P. magdalenae, $P$. orbignyi y $P$. schroederi), actualmente categorizadas con un grado de prioridad de investigación muy alto, considerando la relación con la pesca, el comercio, la distribución y el estadio de conservación, a lo que se suma la escasa información existente (Caldas et al. 2010). En el caso particular de P. magdalenae, su categoría actual es "Near Threatened NT" (IUCN, 2011), al parecer tiene alta representatividad a lo largo de la cuenca del río Magdalena, sus afluentes y en el río Catatumbo, donde habitan enterradas en la arena del fondo de las áreas ribereñas poco profundas y ciénagas (Gutiérrez et al. 2004). No obstante, los individuos de esta especie, capturados incidentalmente por la pesca artesanal en la ciénaga del Sabayo, como en otras localidades (Dahl, 1971) son desechadas, a pesar del aporte nutricional que tienen.
Dada la alta frecuencia y abundancia de la raya de agua dulce $P$. magdalenae en capturas incidentales en la ciénaga de Sabayo (Guaimaral, Magdalena), que contrasta con la falta de información disponible, el presente estudio aporta información pesquera y biológica: abundancia total y relativa, estructura de tallas, talla de madurez sexual, estadio de desarrollo gonadal, fecundidad, índice hepatosomático y gonadosomático, proporción de sexos y factor de condición; además abarca su espectro trófico analizando, cualitativamente, los hábitos alimenticios y sus variaciones espacio temporales, como un primer aporte al conocimiento de la especie en la región.

\section{MATERIALES Y MÉTODOS}

Área de estudio: La ciénaga de Sabayo, se localiza en Guaimaral, corregimiento ubicado en la subregión sur del departamento del Magdalena (9¹8'08' N y 74¹4'10"' O) (Figura 1). Según Villalobos (2006), la ciénaga de Sabayo cuenta con una extensión aproximada de $2 \mathrm{~km}^{2}$ y una profundidad variable (3 a 8m), dependiendo de las épocas climáticas, caracterizadas por presentar dos periodos principales: uno lluvioso, de septiembre a finales de noviembre y uno seco, de noviembre a marzo; posteriormente, se encuentra un periodo menor de lluvias (abril a mayo) y un "veranillo" (mayo a agosto).

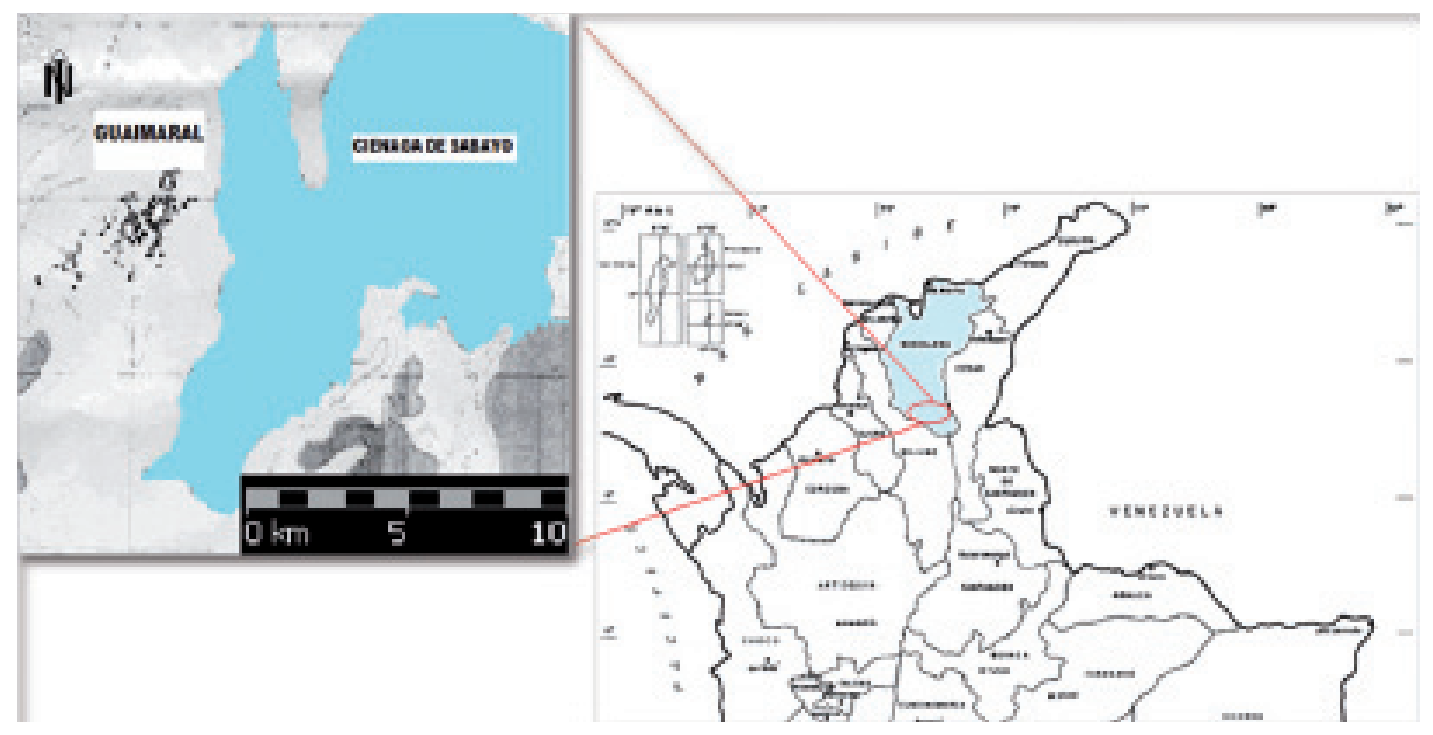

Figura 1. Área de estudio, sector de Guaimaral, Inmediaciones del departamento del Magdalena, Colombia. Modificado de Instituto Geográfico Agustín Codazzi, (1996). 
Fase de campo: Las muestras fueron obtenidas, como pesca incidental de las capturas artesanales, con trasmallo y con chinchorro, las cuales, se analizaron por separado, para los aspectos pesqueros y se integraron, para el análisis del componente biológico. Las muestras procedentes de trasmallo, se tomaron semanalmente, dependiendo de la actividad diaria de los pescadores, durante octubre de 2007 hasta mayo de 2008, extendiendo el arte desde el atardecer hasta la madrugada. El chinchorro empleado, se evaluó a partir de tres faenas efectuadas en marzo y dos en abril y se optó por evaluarlo, debido a la reducida captura de rayas con trasmallo durante la época seca. Una vez obtenida la captura de los dos artes, se separaron los peces óseos de las rayas, estimando, para los primeros, composición y peso total, siendo éste el único registro disponible para el área de estudio.

Fase de laboratorio: Se examinaron animales extraídos por los pescadores artesanales de la región, quienes realizaron sus faenas de pesca cotidianas; no se efectuó pesca de investigación, por lo cual, no se requirió permiso de investigación ni aprobación por parte del Comité de Bioética de la Universidad Jorge Tadeo Lozano, para el procesamiento del material.

Condición reproductiva: La diferenciación sexual, se realizó teniendo en cuenta la descripción de las características internas y externas de los sistemas reproductivos de hembras (estadio de desarrollo de los ovarios, oviductos, útero y glándula nidamental) y de machos (rigidez y rotación de clásper, testículos, epidídimo y vesícula) (Grijalba-Bendeck et al. 2008). El estadio de desarrollo gonadal, se definió según los criterios de Tresierra \& Culquichicón (1993), modificada para $P$. magdalenae. De los embriones, se evaluó número en cada útero, talla y grado de desarrollo, según la escala propuesta por Barbarino E Lasso (2005) y adaptada para el presente estudio.

Espectro trófico: Los estómagos de todos los ejemplares, se fijaron (formalina 4\%) y se conservaron (alcohol 75\%) hasta su posterior análisis en laboratorio. Cada estómago se categorizó, según su grado de llenado y su digestión (Galván et al. 1989; Polo-Silva et al. 2007) y se cuantificó el número y frecuencia de cada componente alimentario contenido (\% $\mathrm{FO})$.

Análisis de información: Aspectos pesqueros: Se calculó la captura por unidad de esfuerzo (CPUE), en número total de individuos y, biomasa, en kilogramos $(\mathrm{kg})$ de $P$. magdalenae mensualmente, para cada arte por separado, estandarizando el tiempo promedio de cada arte, a 1 hora. Los valores obtenidos, se multiplicaron por diez para tener una exposición más clara de sus fluctuaciones; se calculó el error estándar, como medida de su variabilidad y en histogramas de frecuencia, se representó la estructura de tallas por sexos. A manera de información complementaria, se cuantifico la representatividad de la ictiofauna ósea, obtenida durante las faenas.

Aspectos reproductivos: Se evaluaron todos los estadios de desarrollo gonadal y se realizaron descripciones morfológicas de las diferentes etapas de desarrollo gonadal; sin embargo, para la estimación de la talla media de madurez (TMM), se consideraron únicamente las tallas de los machos en estadio III y IV y IIIa, IIIb y IV, en el caso de las hembras, siguiendo los criterios de Tresierra \& Culquichicón (1993) y Arteaga et al. (2004), quienes recomiendan considerar sólo los estados gonadales que ya han alcanzado la madurez para el ajuste de los datos obtenidos a la siguiente función:

$$
P(l)=\left[1 /\left(1+e^{(a+b l)}\right)\right]
$$

Donde: $\mathrm{P}$ es la proporción de individuos maduros, a y b son constantes y l es el intervalo de tallas.

La fecundidad, se halló considerando el número de embriones por útero y el número y promedio de huevos presentes, mensualmente, en cada ovario. Se evaluó la relación entre la talla de las hembras maduras con la talla y número de embriones, según lo propuesto por Babel (1967), a partir de una gráfica de puntos de dispersión. De los embriones, se determinó el peso, el ancho de disco y se tuvo en cuenta la ubicación dentro de los úteros. Se estableció su grado de desarrollo, según Barbarino E Lasso (2005) y modificado para el presente estudio. Debido a que algunos ejemplares llegan sin la cola competa, se tuvo en cuenta la relación peso total y ancho del disco, para calcular el factor de condición, mediante la siguiente ecuación:

$$
\mathrm{FC}=\mathrm{PT} / \mathrm{AD} \text { 。 } 110
$$

Donde: PT es el peso de la raya, $\mathrm{AD}$ es el ancho del disco del individuo $(\mathrm{mm})$ y b, el valor de la relación peso-longitud (Tresierra E Culquichicón, 1993).

Se calculó el Índice Gonadosomático (IGS) y el Hepatosomático (IHS), según las expresiones:

$$
\mathrm{IGS}=(\mathrm{Pg} / \mathrm{Pv}) * 100
$$

Donde: Pg, peso promedio de las gónadas (g) y Pv, peso eviscerado $(g)$.

$$
\mathrm{IHS}=(\mathrm{Ph} / \mathrm{Pv})
$$

Donde: Ph es el peso del hígado (g) y Pv, el peso eviscerado del ejemplar ( $\mathrm{g}$ ). 
Se determinó la proporción sexual, contabilizando el número de machos y de hembras mensualmente y se expresó, en porcentaje, de la siguiente forma:

$$
\% \text { sexo }(\text { machos-hembras })=(\mathrm{Nm} / \mathrm{Nt}) * 100
$$

Donde: Nm, número de machos o hembras y Nt, número total de individuos.

Evaluando la significancia de la proporción obtenida con Chi cuadrado ( $p>0.05$ ) (Tresierra E Culquichicón, 1993). Tanto para adultos, como para embriones, se propuso como hipótesis nula que la proporción hembras y machos era 1:1, tal como ocurre para la mayoría de los vertebrados en la naturaleza.

Aspectos tróficos: La cuantificación en frecuencia de cada componente alimentario, se realizó según Hyslop (1980), teniendo en cuenta las diferencias por tallas:

$$
\% \mathrm{FO}=(\mathrm{Ni} / \mathrm{NT}) * 100
$$

Donde: FO, frecuencia de ocurrencia de cada componente; $\mathrm{Ni}$, número de tractos conteniendo el mismo componente y NT, número de tractos con alimento.

\section{RESULTADOS Y DISCUSIÓN}

En la ciénaga de Sabayo, tanto el chinchorro como el trasmallo, se emplean para capturar peces comerciales. La captura de rayas, se considera como pesca incidental, los peces óseos obtenidos durante el tiempo de muestreo estuvieron representadas por trece especies y la biomasa de estos siempre fue mayor que la de $P$. magdalenae a lo largo de todo el muestreo, las más representativas fueron: el bocachico Prochilodus magdalenae (51\%), pácora - Plagioscion surinamensis (25\%) y el pincho - Cyphocharax magdalenae (9\%), las demás presentaron frecuencias bajas. La ictiofauna acompañante de $P$. magdalenae puede variar de acuerdo al lugar de muestreo en la ciénaga del Sabayo; no obstante, se trata de un cuerpo de agua cerrado, con un sustrato fangoso vegetado, en el que se pueden encontrar otros recursos, como cangrejos (Callinectes spp.), camarones (Paeneus spp. y Macrobranchium), roncos (Bairdiella sp.) y el bagre pintado (Pimelodus grosskopfki), entre otros, que fueron registrados por Yépez E Espinosa (1970), pero no extraídos durante los muestreos realizados.

Se analizaron 488 ejemplares de $P$. magdalenae, 275 machos y 213 hembras; 377 rayas se colectaron empleando trasmallo y 111 con chinchorro. Para el trasmallo, el promedio general de la captura total por unidad de esfuerzo fue
$0,38 \pm 0,06 \mathrm{~kg} / \mathrm{h}$, el mismo promedio para peces óseos fue $0,30 \pm 0,06 \mathrm{~kg} / \mathrm{h}$ y $0,07 \pm 0,02 \mathrm{~kg} / \mathrm{h}$ para rayas. En cuanto al chinchorro, el valor general obtenido fue $81,70 \pm 13,30 \mathrm{~kg} / \mathrm{h}$, los peces óseos presentaron un valor promedio de 55,50 $\pm 13,40 \mathrm{~kg} / \mathrm{h}$ y la captura de rayas se estimó en $26,20 \pm$ $4,60 \mathrm{~kg} / \mathrm{h}$. En términos de número de rayas, el valor promedio de la CPUE para el trasmallo fue 1,43 $\pm 0,37$ ind/h y para el chinchorro 59,30 \pm 9,00ind/h.

Los datos reflejan que la mayor captura de rayas se obtiene con chinchorro en época seca, dado que $P$. magdalenae prefiere fondos de poca profundidad cuando ocurre la disminución en la vegetación acuícola, condiciones que favorecen la captura con este arte, principalmente, entre marzo y abril (Arias, 1993). A medida que se incrementan las lluvias, ocurre el desplazamiento evasivo de las rayas hacia sitios más profundos haciendo ideal el uso del trasmallo, a pesar del incremento de la vegetación acuícola.

La interpretación de estos datos debe ser cuidadosa, debido a que las capturas de peces estimadas para la cuenca Magdalénica no son específicos sobre la inclusión de las especies de rayas, ni se discriminan en biomasa y/o número de individuos; estas capturas oscilan entre 27 y 96kg/día, con un promedio de $47 \mathrm{~kg} /$ día en general para todas las artes de pesca y para el trasmallo, de $3 \mathrm{~kg} / \mathrm{h}$ (Arias, 1993). Para la ciénaga de Sabayo, el promedio de CPUE estimado para el trasmallo fue de $4,00 \pm 0,04 \mathrm{~kg} /$ día y para el chinchorro de $10,00 \pm 3,04 \mathrm{~kg} /$ día; de esta forma, los valores obtenidos en el presente estudio, se pueden considerar representativos y un punto de referencia, debido a que éstos abarcan un área significativamente menor.

El número total de ejemplares analizados $(n=488)$ es alto respecto al evaluado por Teshima \& Takeshita (1992), en un arrastre en el río Magdalena, quienes colectaron 117 especímenes (73 machos y 44 hembras), también con relación al estudio de Ramírez-Gil et al. (2001), en Inírida - Guainía, donde, con diferentes artes de pesca, colectaron 549 ejemplares de $P$. motoro, en 12 meses.

Se capturaron machos con tamaños desde $96 \mathrm{~mm}$ AD hasta $278 \mathrm{~mm}$ y hembras de 82 a $422 \mathrm{~mm}$ AD. En la estructura de tallas, el intervalo mejor representado para las hembras fue 117 a 131, con marca de clase $124 \mathrm{~mm}$, los machos mostraron mayor proporción entre 187 y $201 \mathrm{~mm}$, con marca de clase $194 \mathrm{~mm}$, seguido de 173 a $187 \mathrm{~mm}$, con marca de clase $180 \mathrm{~mm}$. La distribución indicó que las tallas pequeñas tuvieron una elevada frecuencia de captura, lo que puede ser un primer indicio de una presión significativa sobre ejemplares inmaduros, que se puede explicar por el pequeño ojo de malla de los artes que se emplean en la Ciénaga. Por otra parte, se registró como longitudes máximas 278 y 422mm 
AD, para machos y hembras, respectivamente. Yépez E Espinosa (1970) registraron tallas de $380 \mathrm{~mm}$ AD para machos y 560mm AD para hembras, usando chinchorro para su extracción en Venezuela; este aparejo atrapa rayas de mayor tamaño, ya que $P$. magdalenae es denominada una especie bentónica; las hembras alcanzan tallas y pesos máximos mayores que los machos (Yépez E Espinosa, 1970; Puentes et al. 2009), lo que indica que $P$. magdalenae presenta un dimorfismo sexual marcado (Teshima \& Takeshita, 1992; Charvet-Almeida et al. 2005).

Las hembras inmaduras mostraron tallas entre 80 y $175 \mathrm{~mm}$ AD, las hembras en maduración entre 110 y 242mm AD y las maduras fluctuaron de 164 a 422mm AD. Los ovarios de $P$. magdalenae se observaron en todos los estadios, pero el derecho se halló con menor tamaño en hembras maduras (IIla, IIIb y IV) (Figura 2a-c), coincidiendo con la mayoría de las rayas de agua dulce, (Charvet-Almeida et al. 2005) quienes encontraron que solo el ovario izquierdo pareció ser funcional, aunque Teshima $\mathcal{E}$ Takeshita (1992) notaron en $P$. magdalenae que, al parecer, ambos ovarios son funcionales; no se descarta que el ovario derecho está en menor desarrollo o, algunas veces, completamente atrofiado (Figura 2a).

La fluctuación temporal de los estadios de desarrollo fue variable durante el tiempo de estudio: el estadio I fue el mejor representado 49\% ( $n=103)$, seguido por el estadio IIIa con $17 \%$ ( $\mathrm{n}=36)$, posteriormente, el estadio II y IIIb, con $13 \%$ $(n=26) y$, finalmente, el IV, con $8 \%(n=16)$. En este sentido y considerando que $P$. magdalenae en el área de estu-
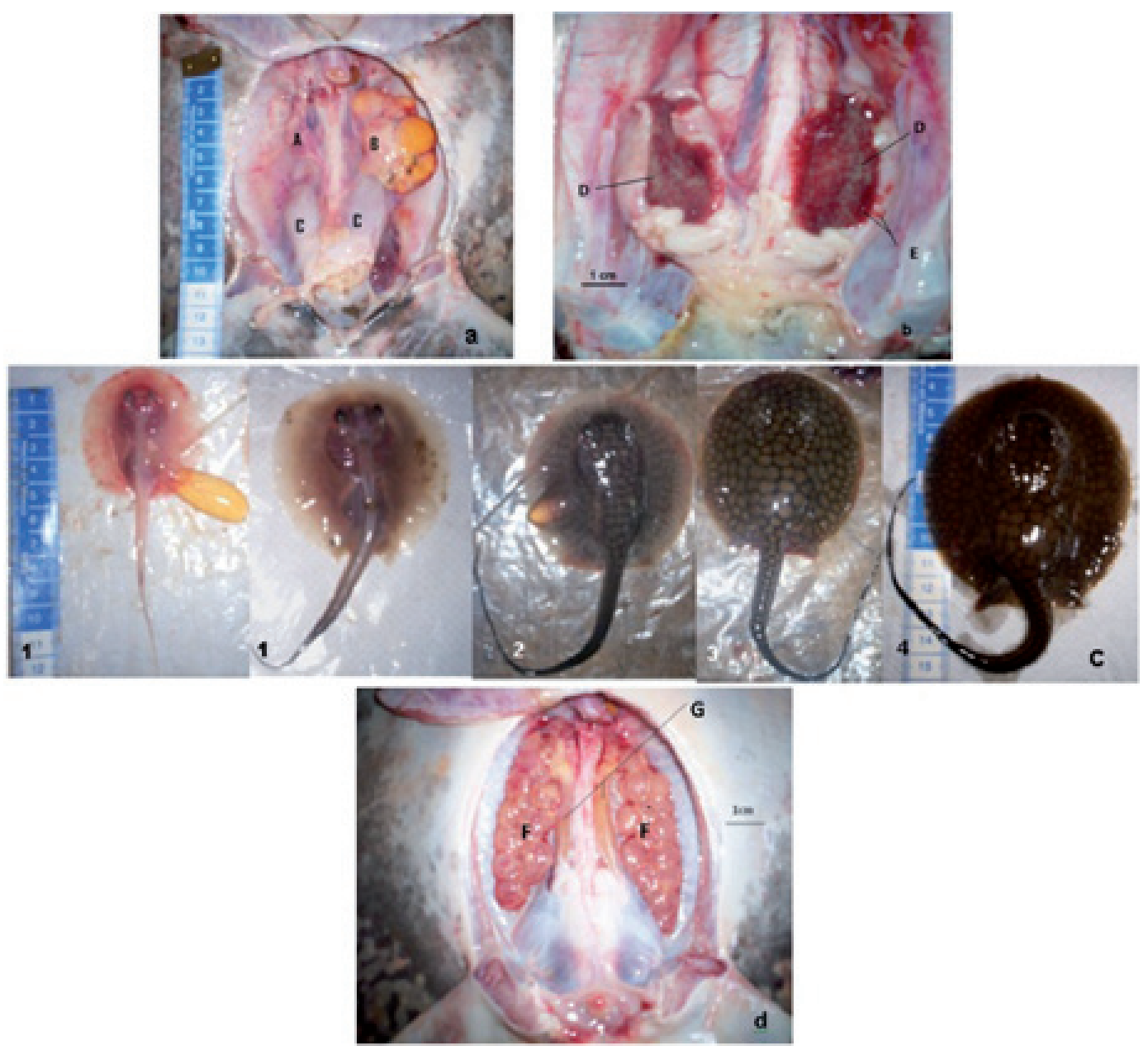

Figura 2. Sistema reproductor de hembras y machos de Potamotrygon magalaenae. a. hembra IIla; b. hembra grávida con los úteros disectados; c. embriones en diferentes estadios de desarrollo; d. testículos de machos maduros. A: Ovario poco desarrollado, B: Ovario funcional, C: Úteros funcionales, D: Leche intrauterina, E: Microvellosidades, F: Testículos maduros y G: Ductos deferentes con epidídimo. 
dio mostró todos los estadios de madurez sexual a lo largo del muestreo, con mayor proporción de hembras maduras en enero y marzo (2008) y gestantes en noviembre (2007), abril y mayo (2008), se propone que su reproducción está determinada por los periodos hidrobiológicos, con una maduración gonadal de cuatro meses, la copulación en épocas secas y de lluvia, con una fecundidad uterina entre tres y cinco embriones, con periodos de gestación entre cuatro a cinco meses en un mismo hábitat. Charvet-Almeida et al. (2005) indican que la reproducción de los potamotrigónidos varía de acuerdo a la especie, donde la maduración gonadal puede estar entre dos y cuatro meses, la copulación en diferentes épocas, la gestación desde tres hasta nueve meses, con nacimientos en época de lluvia, seca o la transición de éstas. Por su parte, Castex (1963), sugiere para estas rayas un periodo reproductivo que presenta estadios definidos; maduración gonadal, copulación, embarazo y nacimiento en un mismo hábitat.

Se registraron 47 embriones procedentes de 24 hembras con tallas entre 175 y 422mm AD; nueve de ellos, se encontraron en desarrollo temprano (estado 1) (30 a 55mm AD), 12 (58 a 65mm AD) poco desarrollados, pero diferenciadas sus principales estructuras (estado 2), 16 (70 a 86mm AD) con alto contenido de saco vitelino (estado 3) y 11 (87 a 95mm AD), con todas sus estructuras bien desarrolladas (estado 4). Los embriones se localizaron en ambos úteros, los cuales, nacen con restos de saco vitelino sin ninguna conexión con la madre; los úteros presentan vellosidades internas en las paredes, siendo más agregadas y notables en estadios maduros (Figura 2b), presentado un modo reproductivo descrito por varios autores (Thorson et al. 1983; Teshima E Takeshita, 1992; Charvet-Almeida et al. 2005) como vivíparo matrotrófico con desarrollo de trofonemata. La talla de nacimiento, se estimó entre 87-95 mm AD, entre tres y cinco neonatos, lo que difiere con lo encontrado por Teshima \& Takeshita (1992), quienes describieron que hembras (210-240mm AD) de $P$. magdalenae desarrollan un simple embrión (42-68mm AD) en cada útero (Figura $2 \mathrm{c}$ ). El dimorfismo sexual, en cuanto a la anchura del disco en hembras que logran mayor tamaño, podría ser una tentativa de maximizar el espacio requerido para la generación de más embriones (Charvet-Almeida et al. 2005), pero en $P$. magdalenae, se observó que la talla materna no determina el número de embriones que estas pueden contener; así, hembras de gran tamaño o pequeñas, pero maduras tienen el mismo número de embriones en los úteros.

Los machos de $P$. magdalenae estuvieron representados por el estadio III, con 49\% ( $n=134)$, seguido del estadio I, con $30 \%$ ( $\mathrm{n}=82$ ) y, finalmente, el estadio II, con un $21 \%$ ( $\mathrm{n}=$ 57); su mayor abundancia se observó con la presencia permanente de individuos maduros a lo largo del muestreo, con mayor proporción en enero (73\%) y abril (66\%) y en menor representatividad en marzo (27\%). Al igual que $P$. aiereba y $P$. orbignyi en los llanos de Venezuela, según Lasso et al. (1996), los machos de $P$. magdalenae no tienen una época reproductiva definida, ya que estuvieron al parecer activos sexualmente durante todo el tiempo de estudio y la cópula tiene lugar durante todo el año.

Al analizar los datos y la fluctuación de la proporción sexual en el tiempo y se comprobó que no hubo segregación de sexos; la proporción de sexos fue 1:1, coincidiendo con lo hallado por Lasso et al. (1996), para Paratrygon aiereba 1:1, pero difiere de lo propuesto para Potamotrygon orbignyi 1:2, en el mismo estudio; luego, Barbarino $\mathcal{E}$ Lasso (2005) registraron para $P$. aiereba una proporción de 1:2 machos a hembras, siendo diferente a lo apreciado por Lasso et al. (1996) en esta especie; es probable que la proporción pueda variar con respecto al arte usado, ya que Barbarino $E$ Lasso (2005) emplearon arpón, más común para la captura de esta especie en Venezuela y altamente selectivo, ya que busca atrapar especímenes de mayor tamaño, como las hembras. En cuanto al trasmallo y el chinchorro, son artes que no van dirigidos a las rayas, por lo que la captura es incidental, obteniendo especímenes de ambos sexos y de diferentes tallas en similares proporciones.

Las hembras presentaron una talla media de madurez de $240 \mathrm{~mm}$ AD y los machos de $202 \mathrm{~mm}$ AD, obtenida a partir de 77 hembras maduras (IIla, IIlb y IV) y 132 machos maduros (III). La talla mínima de madurez fue de $164 \mathrm{~mm} \mathrm{AD}$, en hembras y $160 \mathrm{~mm}$ AD, en machos (Figura 3). Thorson et al. (1983) hallaron en $P$. circularis hembras que ya empiezan su maduración a los 350 hasta $450 \mathrm{~mm}$ AD y para $P$. motoro ocurre entre 240 - 320mm AD; los resultados obtenidos de la talla mínima y media de madurez indican que las hembras de $P$. magdalenae empiezan su maduración entre 164 - 240mm AD y los machos entre 160 - 210mm AD.

A diferencia de lo descrito por González E Oyarzún (2002), quienes señalan que el IGS se relaciona en forma inversa al FC y al IHS, debido a una demanda de energía por parte del pez para procesos reproductivos, estos índices en $P$. magdalenae no revelaron cambios a lo largo de los meses. Se observó que no existe una correspondencia entre IGS y FC en hembras $\left(r^{2}=0,305 ; p=0,46\right)$, tampoco en machos $\left(r^{2}=\right.$ $0,279 ; p=0,50)$. Por tanto, en la raya de río no se evidencia un comportamiento que pudiera estar reflejando movimientos de energía somática hacia las gónadas. Lo mismo sucede con la relación entre el IGS e IHS, en hembras y machos $\left(r^{2}=0,050 ; p=0,89\right.$ y r ${ }^{2}=0,22 ; p=0,59$, respectivamente), que tampoco mostró una concordancia, lo que estaría indicando continuo flujo de energía del hígado hacia las 


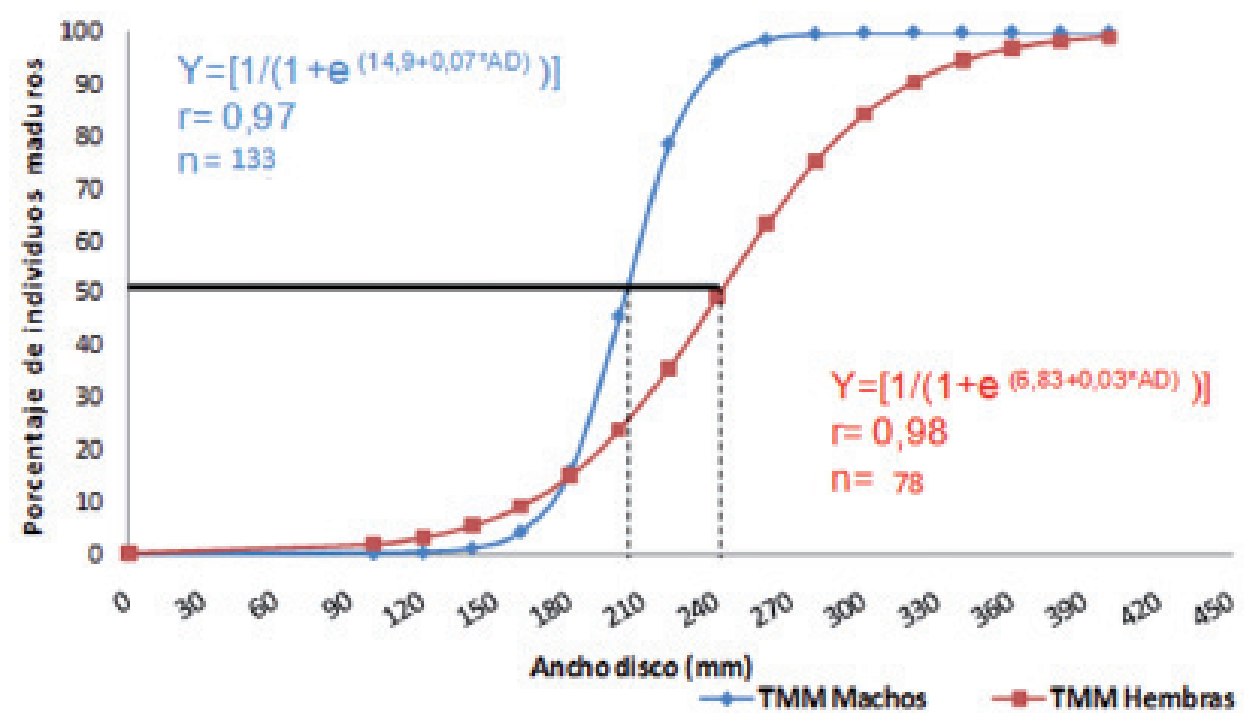

Figura 3. Talla media de madurez para las hembras y para los machos de Potamotrygon magdalenae, la curva representa el ajuste de los datos.

gónadas, utilizada para la producción de gametos, sin estar centrado en un período específico. Al aparecer $P$. magdalenae es una especie con un comportamiento de alimentación constante, lo que podría estar indicando, en alguna medida, un aporte constante de energía, que le permitirían soportar la maduración y el desove. Esto también la anotan Teshima $\varepsilon$ Takeshita (1992), indicando que la especie puede no tener una estación reproductiva definida, presentándose machos y hembras maduras todo el año.

En los ocho meses de muestreo, se analizaron 266 estómagos: 154 procedentes de machos $(57,8 \%)$ y 112 de hembras (42,1\%); 39, (14,6\%) estuvieron vacíos y $227,(85,3 \%)$ tenían algún tipo de contenido. El grado de llenado estomacal se encontró, principalmente, representado por la escala $1,(1 / 4$ de llenado), con un $30 \%$, seguido de la escala 2 , (1/2 de llenado), con un $21,8 \%$ de estómagos y, finalmente, las escalas 4 (lleno totalmente), 3 ( $3 / 4$ de llenado) y 0 (vacío), con porcentajes de llenado similares, $17,3 \%, 15,4 \%$ y $15,4 \%$, respectivamente, indicando, para el último valor, que los especímenes se habían alimentado mucho antes de su captura (Machado et al. 2004). En el total de estómagos analizados predominó el grado de digestión 4 (presencia de materia orgánica y restos de esqueleto), con un $30,3 \%$, seguido del grado 1 (individuos que presentan todas las características morfológicas completas que los hacen fácilmente identificable), con un $28,3 \%$ y 2 (ejemplares con cabeza y ojos, sin piel y músculos al descubierto), con $21,6 \%$, siendo el estadio 3 (presas sin cabeza, algunas partes del cuerpo presentes y esqueleto axial) el menos frecuente.
Los contenidos estomacales, se agruparon en tres ítems: insectos acuáticos (Ephemeroptera: Polymitarcyidae y sus larvas, seguidos por Diptera: Ceratopogonidae, Chironomidae y sus larvas, Odonata: Aeshnidae y huevos en general), moluscos (opérculos y vísceras de gasterópodos) y detritus (partículas de sedimento y materia orgánica no identificada).

La familia Polymitarcyidae, en general, presentó la mayor frecuencia de ocurrencia de todos los componentes hallados, con un $(55,6 \%)$, seguido del detritus $(30,8 \%) \mathrm{y}$, finalmente, aparecieron larvas (35,7\%); la menor fue registrada para las vísceras de gasterópodos $(1,5 \%)$ y la familia Aeshnidae y Chironomidae (2,6\%). La frecuencia de aparición de Polymitarcidae en los contenidos estomacales puede ser explicada por una alta abundancia en la ciénaga del Sabayo, donde se encuentran las condiciones propicias para desarrollarse, como aguas cálidas y fondos arenosos; son un grupo bentónico abundante empleado como indicador de aguas limpias (Roldán, 1988). Por su parte, Ceratopogonidae es una familia común en aguas lénticas, charcas y lagos con material orgánico en descomposición, cuya presencia y abundancia se suele interpretar como indicador de aguas mesoeutróficas (Roldán, 1988); su aparición junto con la familia Aeshnidae, como presa de las rayas se explica, porque suelen estar asociados a las raíces de macrófitas (Roldán, 1988), muy abundantes en el Sabayo.

Resultados similares fueron propuestos por Dahl (1971), quien informa para $P$. magdalenae que su alimento se basa 
de gusanos, de moluscos y de otros invertebrados. El espectro trófico descrito es similar al propuesto para P. orbignyi, el cual, está conformado por 13 categorías de alimento, entre las que se encuentra Coleoptera, Hemiptera, Odonata, Trichoptera, Chironominae, Ceratopogonidae, ostrácodos, plantas e insectos sin identificar (Lasso et al. 1996). La composición de los ítems, tanto en hembras como en machos de diferentes tallas, estuvo representada por insectos acuáticos de actividad bentónica en cuerpos de aguas lentas, coincidiendo con lo que citan Galvis et al. (1997), quienes argumentan que $P$. magdalenae prefiere los fondos lodosos con aguas turbias y poco profundas, consumiendo estadios larvarios de insectos y de detritus. Con base en los resultados y en lo antes mencionado, se confirma que $P$. magdalenae en la ciénaga de Sabayo es consumidora primaria, insectívoradetritívora de hábitos bentónicos, como lo propone Villa-Navarro (1999), aunque el presente estudio indicó que la mayor parte de los estómagos analizados contuvieron escaso alimento digerido y que la materia orgánica hallada (detritus), probablemente, está relacionada con la ingestión accidental de estas partículas (Machado et al. 2004), al succionar para capturar sus presas, como lo registraron en $P$. orbignyi y $P$. iwame, Lasso et al. (1996).

Analizando la dieta de la especie en función del microhábitat ocupado, se sugiere que $P$. magdalenae tiene una dieta especializada consumiendo más Polymitarcidae, Ceratopogonidae y larvas de estos, sin encontrar diferencias temporales ni espaciales de estos recursos, a diferencia de lo que proponen Silva E Uieda, (2007) en P. motoro, quienes encontraron una dieta especializada sobre Ephemenoptera para esta especie, distinta de $P$. falkneri, para la cual, propusieron una dieta generalizada, conformada por Mollusca, Hemiptera y Trichoptera.

Agradecimientos: Este trabajo se realizó con el apoyo logístico y financiero de la Universidad de Bogotá Jorge Tadeo Lozano (UJTL), Facultad de Ciencias Naturales e Ingeniería, Programa de Biología Marina, Sede Santa Marta. Agradecimientos especiales a los pescadores de la ciénaga del Sabayo y Guaimaral. Contribución 028 del Grupo de Investigación de Peces del Caribe (GIPECA-UJTL). Conflicto de intereses: Este manuscrito fue preparado y revisado con la participación de todos los autores, quienes declaramos que no existe ningún conflicto de intereses que ponga en riesgo la validez de los resultados presentados.

\section{BIBLIOGRAFÍA}

1. ARIAS, P. 1993. Artes y métodos de pesca en aguas continentales de Colombia. Subgerencia de operaciones.
Primera edición. Instituto Nacional de Pesca y Acuicultura INPA. Bogotá, 39p.

2. ARTEAGA, E.; CRIALES-HERNÁNDEZ, M.I.; RODRÍGUEZ D.J.; GÓMEZ-CANCHONG P.; MANJARRÉS, L. 2004. Dinámica reproductiva de los stocks de pargo rayado Lutjanus synagris (Linnaeus, 1758) en el área norte del Caribe colombiano. En: Manjarrés, L. (Ed). 2004. Pesquerías demersales del área norte del Mar Caribe de Colombia y parámetros biológico - pesqueros y poblacionales del recurso pargo. Instituto Nacional de Pesca y Acuicultura INPA - Instituto Colombiano para el Desarrollo de la Ciencia y la Tecnología "Francisco José de Caldas" - COLCIENCIAS. p.203-215.

3. BABEL, J.S. 1967. Reproduction, life history, and ecology of the round stingray Urolophus halleri Cooper. Calif. Dep. Fish Game, Fishery Bulletin. p.137, 104.

4. BARBARINO, A.; LASSO, C. 2005. Pesquería y aspectos biológicos de la raya manta Paratrygon aiereba (Müller y Henle, 1841) (Myliobatiformes: Potamotrygonidae), en el río Apure, Venezuela. Memoria de la Fundación La Salle de Ciencias Naturales 163:93-108.

5. CALDAS, J.P.; CASTRO-GONZÁLEZ, E.; PUENTES, V.; RUEDA, M.; LASSO, C.; DUARTE, L.O.; GRIJALBABENDECK, M.; GÓMEZ, F.; NAVIA, A.; MEJÍA-FALLA, P.; BESSUDO, S.; DIAZGRANADOS, M.C.; ZAPATA, L. (Eds.). 2010. Plan de Acción Nacional para la Conservación y Manejo de Tiburones, Rayas y Quimeras de Colombia (PAN-Tiburones Colombia). Instituto Colombiano Agropecuario, Secretaría Agricultura y Pesca San Andrés Isla, Ministerio de Ambiente, Vivienda y Desarrollo Territorial, Instituto de Investigaciones Marinas y Costeras, Instituto Alexander von Humboldt, Universidad del Magdalena, Universidad Jorge Tadeo Lozano, Pontificia Universidad Javeriana, Fundación SQUIALUS, Fundación Malpelo y otros Ecosistemas Marinos, Conservación Internacional, WWF Colombia. Editorial Produmedios, Bogotá. 60p.

6. CASTEX, M. 1963. Observaciones sobre la raya de río Potamotrygon motoro (Müller y Henle). Ministerio de Educación y Justicia de la Nación. Dirección General de Cultura. Hidrobiología. Tomo I. Buenos Aires, 2; 17p.

7. CHARVET-ALMEIDA, P.; GOES, M.; PINTO, M. 2005. Reproductive aspects of freshwater stingrays (Chondrichthyes: Potamotrygonidae) in the Brazilian Amazon Basin. J. Northwest Atlantic Fishery Science. Brasil, 35:165-171. 
8. DAHL, G. 1971. Los peces del Norte de Colombia. Ministerio de Agricultura. Instituto de desarrollo de los recursos naturales renovables INDERENA, Bogotá, $391 \mathrm{p}$.

9. GALVÁN, F.; NIENHUIS, H.; KLIMLEY, P. 1989. Seasonal abundance and feeding habits of shark of the lower gulf of California, Mexico. California Fish and Game. 75:74-84.

10. GALVIS, G.; MOJICA, J.; CAMARGO, M. 1997. Peces del Catatumbo. Asociación Cravo Norte (ECOPETROL, OXY, Shell). $1^{\text {a }}$ ed. Bogotá, p.24-25.

11. GONZÁLEZ, P.; OYARZÚN, C. 2002. Variabilidad en índices biológicos en Pinguipes chilensis (Perciformes, Pinguipedidae): ¿Están realmente correlacionados? Gayana. 66(2):249-254.

12. GRIJALBA-BENDECK, M.; ACERO, A.; GONZÁLEZ, E. 2008. Biología reproductiva de Rhinobatos percellens (Walbaum, 1792) (Batoidea: Rajiformes) en el Caribe colombiano. Rev. Biología Marina y Oceanografía. 43(3):469-481.

13. GUTIÉRREZ, J.; BENÍTEZ-GUERRA, I.; BENÍTEZGUERRA, G. 2004. Envenenamiento por rayas de agua dulce. Rev. Facultad de Medicina. 27(2):131-134.

14. HYSLOP, E. 1980. Stomach contents analysis: A review of methods and their application. J. Fish Biology. 17:411429.

15. IUCN. 2011. IUCN Red List of Threatened Species. Version 2011.1. Disponible desde Internet en: www. iucnredlist.org (con acceso 03/08/11).

16. LASSO, C.; RIAL, A.; LASSO-ALCALÁ, O. 1996. Notes on the biology of the freshwater stingrays Paratrygon aiereba (Müller y Henle, 1841) and Potamotrygon orbignyi (Castelnau, 1855) (Condrichthyes: Potamotrygonidae) in the Venezuelan Llanos. Aqua, J. Ichthyology and Aquatic Biology. 2(3):39-50.

17. MACHADO, A.; CHARVET-ALMEIDA, P.; BORGES, R. 2004. Preliminary observations on the feeding of the freshwater stingrays Potamotrygon orbignyi, Potamotrygon scobina and Plesiotrygon iwamae (Chondrichthyes: Potamotrygonidae) in the Cotijuba Island region - Pará - Brazil. EN: Biology and Conservation of Freshwater Elasmobranchs. Symposium Proceedings. International Congress on the Biology of Fish. p.41-51.
18. NELSON, J. 2006. Fishes of the world. Forth Edition. Department of Biological Science, University of Alberta. John Wiley and Sons, Inc. Canada. 601p.

19. POLO-SILVA, C.; BAIGORRÍ-SANTACRUZ, A.; GALVÁNMAGAÑA, F.; GRIJALBA-BENDECK, M.; SANJUANMUÑOZ, A. 2007. Hábitos alimentarios del tiburón zorro Alopias superciliosus (Lowe, 1839), en el Pacífico ecuatoriano. Rev. Biol. Marina y Oceanogr. 42(1):5969.

20. PUIENTES, V.; NAVIA, A.; MEJÍA-FALLA, P.; CALDAS, J.; DÍAZGRANADOS, M.; PADILLA, L. 2009. Avances en el conocimiento de tiburones, rayas y quimeras de Colombia. Fundación SQUALUS, Ministerio de Ambiente Vivienda y Desarrollo Territorial, Instituto Colombiano Agropecuario, COLCIENCIAS, Conservación Internacional, WWF Colombia. p.195245.

21. RAMÍREZ-GIL, H.; CARRILLO-VILLAMAR, L.; LACERPADILLA, E.; AJIACO-MARTÍNEZ, R. 2001. La pesca de especies de interés ornamental en el área de influencia de Puerto Carreño. En: Ramírez, H.; Ajiaco, R. eds. La pesca en la baja Orinoquia colombiana: una visión integral. $1^{\mathrm{a}}$ ed. Instituto Nacional de Pesca y Acuicultura. p.123-137.

22. ROLDÁN, G. 1988. Guía para el estudio de los macroinvertebrados acuáticos del Departamento de Antioquia. Fondo FEN Colciencias, Colciencias, Universidad de Antioquia. 217p.

23. SILVA, T.; UIEDA, V. 2007. Preliminary data on the feeding habits of the freshwater stingrays Potamotrygon falkneri and Potamotrygon motoro (Potamotrigonidae) from the Upper Paraná River basin, Brazil. Biota Neotropica 7(1):221-226.

24. TESHIMA, K.; TAKESHITA, K. 1992. Reproduction of the freshwater stingray Potamotrygon magdalenae taken from the Magdalena river system in Colombia, South America. Bull. Seikai Nal Fisheries Res. Inst.. 70:11-27.

25. THORSON, T.; LANGHAMMER, J.; OETINGER, M. 1983. Reproduction and development of the South America freshwater stingrays, Potamotrytgon circularis and P. motoro. Environ. Biol. Fishes. 9(1):3-24.

26. TRESIERRA, A.; CULQUICHICÓN, Z. 1993. Biología pesquera. Consejo Nacional de Ciencia y Tecnología, Perú. 432p. 
27. VILLA-NAVARRO, F. 1999. Estudio biológico-pesquero de la represa de Prado, para la determinación de las especies promisorias para acuicultura. Universidad del Tolima, Cortolima. Colombia. 105p.

28. VILLALOBOS, U. 2006. Génesis de Guaimaral. Editorial Imaginaria. Colombia. p.28-30.
29. YÉPEZ, A.; ESPINOSA, V. 1970. Observaciones en el peso y ancho del disco de la raya pintada Potamotrygon magdalenae (Duméril). Acta Scientífica. 8(2):7-10.

Recibido: Febrero 18 de 2011

Aceptado: Septiembre 12 de 2011 